\title{
Oxygen temperature anisotropy and solar wind heating above coronal holes out to $\mathbf{5} \boldsymbol{R}_{\odot}$
}

\author{
D. Telloni ${ }^{1}$, E. Antonucci ${ }^{1}$, and M. A. Dodero ${ }^{2}$ \\ 1 Istituto Nazionale di Astrofisica (INAF), Osservatorio Astronomico di Torino, Strada Osservatorio 20, 10025 Pino Torinese, Italy \\ e-mail: telloni@to.infn.it \\ 2 University of Torino, via P. Giuria 1, 10125 Torino, Italy
}

Received 18 April 2007 / Accepted 12 September 2007

ABSTRACT

\begin{abstract}
The purpose of the paper is to measure the degree of temperature anisotropy of the oxygen ions in the outer corona. The ratio of the Doppler dimmed OVI 1037-1032 line intensity as a function of the velocity of the fast solar wind, computed for typical values of coronal density, is consistent with the observed ratio, only when a significant temperature anisotropy is established in polar coronal holes. The oxygen ion velocity distribution is constrained to be bi-Maxwellian from $2 R_{\odot}$ to $3.7 R_{\odot}$, where the lowest degree of anisotropy compatible with the observational data increases up to $\sim 7$ at $2.9 R_{\odot}$, proving that the oxygen ions are accelerated across the magnetic field, in accordance with a preferential energy deposition perpendicular to the field lines, consistent with the process of ion-cyclotron dissipation of Alfvén waves. The most plausible evolution of the velocity distribution of the $\mathrm{O}^{+5}$ ions departs from the bi-Maxwellian configuration at $2 R_{\odot}$, according to an anisotropy ratio that reaches its maximum value $T_{\perp} / T_{\|} \sim 14$ at $2.9 R_{\odot}$, and further out approaches isotropy, at $3.7 R_{\odot}$. In response to the acceleration across the field, energy redistribution along the magnetic field lines accelerates the oxygen component of the solar wind to velocities of $760 \mathrm{~km} \mathrm{~s}^{-1}$ at $5 R_{\odot}$. The variation of the anisotropy ratio with the heliocentric distance might be satisfactorily explained by theoretical models of the fast solar wind heating based on the oxygen cyclotron instability or the fast shock mechanism. The observations of the extended corona analyzed in this paper are performed with the Ultraviolet Coronagraph Spectrometer on board the Solar Heliospheric Observatory, during the solar minimum activity period 1996-1997.
\end{abstract}

Key words. Sun: corona - Sun: UV radiation - Sun: solar wind

\section{Introduction}

Spectroscopic observations of the polar coronal holes performed with the Ultraviolet Coronagraph Spectrometer (UVCS) on board the Solar and Heliospheric Observatory (SOHO) have revealed preferential heating of the $\mathrm{O}^{+5}$ ions across the magnetic field in the regions where the fast wind is accelerated (Antonucci 1998; Kohl et al. 1998; Li et al. 1998; Dodero et al. 1998; Wilhelm et al. 1998; Cranmer et al. 1999). This phenomenon is likely to be ascribed to ion-cyclotron resonance scattering by high frequency Alfvén waves (Kohl et al. 1998; Cranmer et al. 1999). The process leads to the formation of a strong temperature anisotropy at least beyond $1.8 R_{\odot}$, with an anisotropy ratio $10<T_{\perp} / T_{\|}<100$. The topic has been recently reviewed by Antonucci (2006) and Kohl et al. (2006).

Considerable efforts have been devoted to proposing models of energy deposition based on wave-particle interaction processes that can explain the extremely large temperature anisotropy ratio $(\sim 100)$ derived from the UVCS data. However, the observational data are also consistent with a polar region where the oxygen temperature across the magnetic field at maximum is 10-20 times larger than that along the field lines (Lee \& Wu 2000; Vocks \& Marsch 2002; Cranmer \& van Ballegooijen 2003; Antonucci et al. 2000, 2004; Hellinger et al. 2005; Li \& Habbal 2005).

Recently the anisotropy of the oxygen ion velocity distribution has been questioned (e.g. Raouafi \& Solanki 2004, 2006), in a paper that has highlighted the contribution of the Doppler effects due to the outflow velocity of the emitting ions when reconstructing the profile of the OVI lines emitted by a model corona.

The paper by Telloni et al. (2007), however, has evaluated the relevance of this effect and has clearly shown that the Doppler shifts due to the expansion of the corona do not significantly affect the results on the temperature anisotropy established in the outer corona, thus confirming the interpretation of the data proposed up to now by the UVCS team. These results have allowed us to determine the altitude and thickness of the coronal layer where the oxygen ion velocity distribution has to be anisotropic and, in turn, where the energy is deposited more efficiently across the magnetic field.

In this paper, a detailed analysis of the degree of anisotropy in the oxygen ion velocity distribution in polar coronal holes out to $5 R_{\odot}$ is performed, with the intent to further constrain the value of the anisotropy ratio. Possible scenarios for the variations of the anisotropy ratio with increasing heliocentric distance, likely to be related to the efficiency of the deposition of the energy across the magnetic field and its redistribution along the field itself, are discussed.

\section{Observations and diagnostic method}

The aim of this study is to constrain the value of the anisotropy degree in the oxygen ion velocity distribution in the coronal regions where the fast wind is accelerated, which are characterized by an extremely large oxygen temperature across the magnetic field lines. The data analyzed consist of coronal hole 
Table 1. Observations performed with UVCS.

\begin{tabular}{lll}
\hline \hline Date & Height $\left(R_{\odot}\right)$ & Pole \\
\hline November 10, 1996 & 3.0 & North \\
January 9-15, 1997 & 3.1 & North \\
April 14-20, 1997 & $3.1-4.2$ & South \\
June 7-8, 1997 & $2.9-3.1$ & North \\
October 18-19, 1997 & $1.4-3.8$ & North \\
December 20-22 31, 1997 & $1.4-5.0$ & South \\
\hline
\end{tabular}

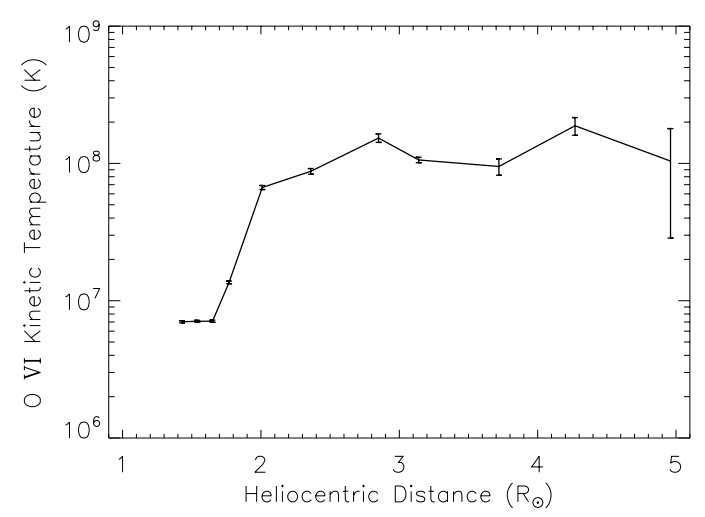

Fig. 1. O VI kinetic temperature results as a function of the heliocentric distance.

observations performed with the UVCS, from 1996 November to 1997 December in the last solar minimum activity period. The regions scanned by the coronagraph are coronal holes present both at the North and South pole between $1.4 R_{\odot}$ and $5 R_{\odot}$, depending on the observation data set. In Table 1 the date and region of the observations are shown.

The broadening of the OVI coronal lines provides information on the oxygen temperature perpendicular to the magnetic field lines, which at the poles roughly coincide with the radial direction, by probing the ion velocity distribution along the line of sight. The width of the OVI 1032 line is derived according to the procedure described in Telloni et al. (2007), accounting for the line broadening due to the Doppler effect produced by the outflow velocity of the emitting ions. The kinetic temperature of the $\mathrm{O}^{+5}$ ions, $T_{k, \mathrm{OVI}}$, including the effect of both thermal and nonthermal plasma motions, is inferred directly from the line width:

$T_{k, \mathrm{OVI}}=\left(\frac{\sigma c}{\lambda_{0}}\right)^{2} \frac{m_{\mathrm{O}}}{k_{\mathrm{B}}}$,

where $c$ is the speed of light, $k_{\mathrm{B}}$ is the Boltzmann constant, and $m_{\mathrm{O}}$ is the oxygen mass.

The oxygen kinetic temperature results, averaged over intervals of the data sample, as a function of the heliocentric distance are shown in Fig. 1.

The oxygen kinetic temperature shows that the ion velocity distribution along the line of sight remains extremely high in an extended region of the solar corona. The kinetic temperature increases up to $\sim 1.5 \times 10^{8} \mathrm{~K}$ at $2.9 R_{\odot}$ and somehow flattens beyond this height. The large increase of the oxygen kinetic temperature with height and its extremely high values achieved above $2 R_{\odot}$ indicate undoubtedly that the oxygen ion velocity distribution is anisotropic across the coronal magnetic field, at least beyond this altitude. An excess of width of the velocity distribution along the line of sight is a clear indication of preferential acceleration of the oxygen component of the fast wind across the magnetic field, since this effect can only be due to a vigorous energy deposition process acting in the outer corona in that direction. We

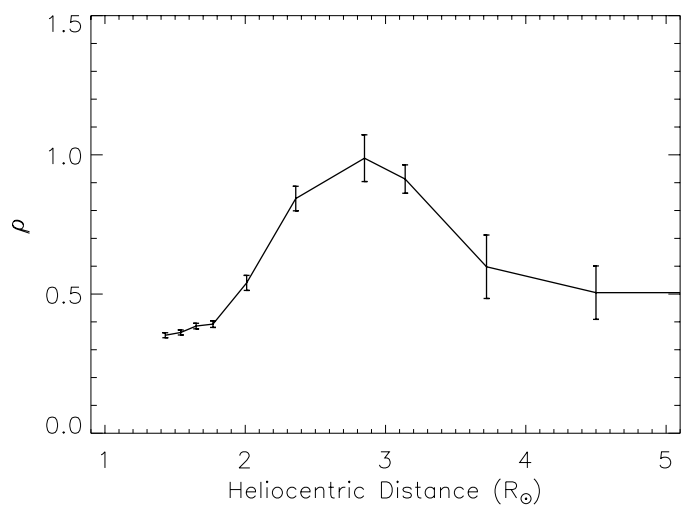

Fig. 2. OVI 1037-1032 line intensity ratio $\rho$ as a function of the heliocentric distance.

expect energy deposition already in the inner corona (probably due to different mechanisms such as magnetic reconnection, turbulence, wave dissipation and plasma instabilities) in order to explain the oxygen temperature of $7 \times 10^{6} \mathrm{~K}$ observed at $1.4 R_{\odot}$. However, the sharp increase in the oxygen kinetic temperature further out at $1.8 R_{\odot}$ is likely to be due to either a different energy deposition process or an abrupt change in its efficiency. An almost steady state is then established above $2.9 R_{\odot}$, where the oxygen temperature flattens with fluctuations around its maximum value of about $10^{8} \mathrm{~K}$.

The spectroscopic diagnostic based on the Doppler dimming effect, developed by Noci et al. (1987), is applied to derive the speed of the oxygen component of the solar wind. In the regions where the OVI line broadening is extremely large, beyond $2 R_{\odot}$, by using this diagnostic it is possible to derive some information on the oxygen ion velocity distribution along the magnetic field lines.

In the expanding outer corona, the total emission of the O VI lines consists of the collisional component and the radiative one: their relative importance varies with the heliocentric distance and only the resonantly scattered component, due to the exciting spectrum emitted from the lower atmosphere, is affected by Doppler dimming. The intensity ratio of the OVI doublet can be used to study the dynamical properties of the coronal regions where the solar wind is accelerated, since the two lines are differently affected by Doppler dimming, which increases with the outflow velocity of the corona. Therefore, the speed of the expansion of the coronal plasma above polar holes is derived by comparing the expected O VI line intensity ratio, inferred by employing an analytical model of the solar corona consistent with the observations at the minimum of activity, with the experimental ratio, derived from the data. The observed OVI 10371032 line intensity ratios, averaged over intervals of the data sample, as a function of the heliocentric distance are shown in Fig. 2.

The integration along the line of sight (LOS) requires the modeling of all the physical quantities that influence the oxygen coronal emission, that is the magnetic field configuration, and the electron density and temperature. The assumptions and models employed in this analysis to derive the minimum degree of anisotropy of the oxygen ion velocity distribution and the corresponding outflow velocity are the same adopted in Telloni et al. (2007). The magnetic model of Banaszkiewicz et al. (1998) is assumed for describing the magnetic field at solar minimum corona. The electron density is that derived by Antonucci et al. (2004), who developed a spectroscopic method that allows the derivation of both the electron density and the outflow velocity 
from UVCS data in a self consistent procedure. The density values inferred in this way are consistent with the model developed by Guhathakurta et al. (1999). The assumed electron temperature is compatible at low altitudes with the measurements by David et al. (1998) and at larger heights with the asymptotic value of $\sim 1 \times 10^{5} \mathrm{~K}$ achieved at $1 \mathrm{AU}$ (e.g. Schwenn 1990).

The integration of the $\mathrm{O}^{+5}$ emissivity along the LOS is performed in a cone centered on the solar axis 60 degrees wide, where a spherical symmetry of the coronal hole is assumed. This assumption is based on the fact that in the magnetic field configuration adopted in the analysis (Banaszkiewicz et al. 1998) the field lines deviate significantly from a radial configuration beyond about 60 degrees.

The theoretical ratio, and thus the inferred outflow speed of the fast solar wind, is strongly dependent on the degree of anisotropy of the oxygen ion velocity distribution, assumed to compute such a ratio. The component of the oxygen temperature perpendicular to the magnetic field lines is approximately the kinetic temperature inferred from the observations, $T_{\mathrm{OVI}, \perp} \sim$ $T_{k, \text { OVI }}$. In the case of isotropy of the oxygen ion velocity distribution, the component of the oxygen temperature parallel to the magnetic field lines is assumed to be equal to the perpendicular one, thus $T_{\mathrm{OVI}, \|}=T_{\mathrm{OVI}, \perp}=T_{k, \mathrm{OVI}}$. In the case of anisotropy, in first approximation the parallel component is here assumed to be varying with the heliocentric distance as the electron temperature. The oxygen temperature parallel to the magnetic field can thus be expressed as:

$T_{\text {OVI, }}(r)=n \cdot T_{\mathrm{e}}(r)$

where $T_{\mathrm{e}}$ is the electron temperature assumed in polar coronal hole regions (Telloni et al. 2007) and $n$ is varied in the analysis from unity (maximum anisotropy) to the maximum allowed value $n_{\max }$ :

$n_{\max }(r)=\frac{T_{k, \text { OVI }}(r)}{T_{\mathrm{e}}(r)}$

The value of $n$ regulates that of the parallel component of the oxygen temperature as a function of the heliocentric distance, and in turn the expected OVI line intensity ratio and the inferred outflow velocity. Figure 3 shows three different curves (as functions of the local outflow velocity and the degree of anisotropy of the oxygen ion velocity distribution) representing the theoretical ratio of the intensity of the OVI lines, compared to the observed ratio.

At $2.9 R_{\odot}$ not all the values of the anisotropy degree yield the expected O VI doublet line intensity ratio consistent with the observed one (lower continuous curve in the figure). The degree of anisotropy found when the theoretical line intensity ratio curve is tangent to the experimental value (dashed curve in the figure) is the lowest one allowed by observations. Thus, the Doppler dimming analysis of the OVI spectral lines can only constrain the lower limit of the anisotropy degree in the oxygen ion velocity distribution, since only the lowest temperature anisotropy ratio consistent with the observations can be inferred.

The lowest degree of anisotropy is estimated at each heliocentric height where the isotropy of the oxygen velocity distribution is not allowed. Thus, the maximum value of the oxygen temperature parallel to the magnetic field lines as a function of the heliocentric distance can be derived. The analysis is then iterated since the derived parallel component of the oxygen temperature is not necessarily the same as the one assumed in first approximation (Eq. (2)). The iterative procedure is performed until input and output values of the lowest degrees of anisotropy,

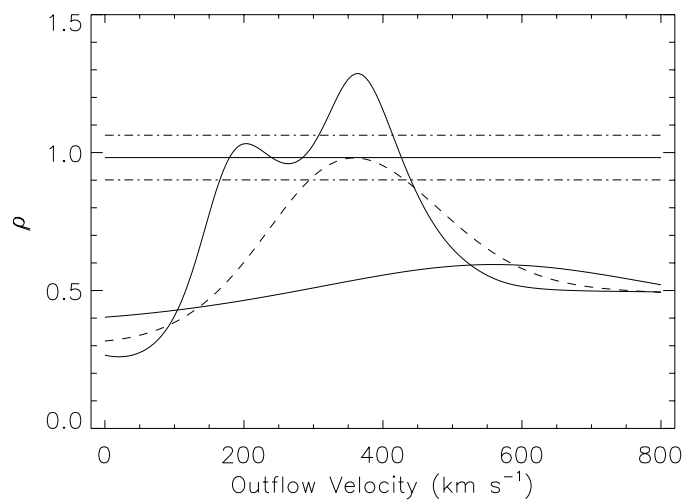

Fig. 3. O VI 1037-1032 line intensity ratio at $2.9 R_{\odot}$ computed for a parallel component of the oxygen ion $T_{\|, \min }=T_{\mathrm{e}}(r)$ (upper continuous curve, maximum anisotropy) and $T_{\|, \max }=T_{\mathrm{k}, \mathrm{OVI}}(r)$ (lower continuous curve, isotropy); the dashed curve, tangent to the observed OVI line intensity ratio (horizontal line), is computed for the lowest degree of anisotropy of the oxygen ion velocity distribution allowed by the observations.

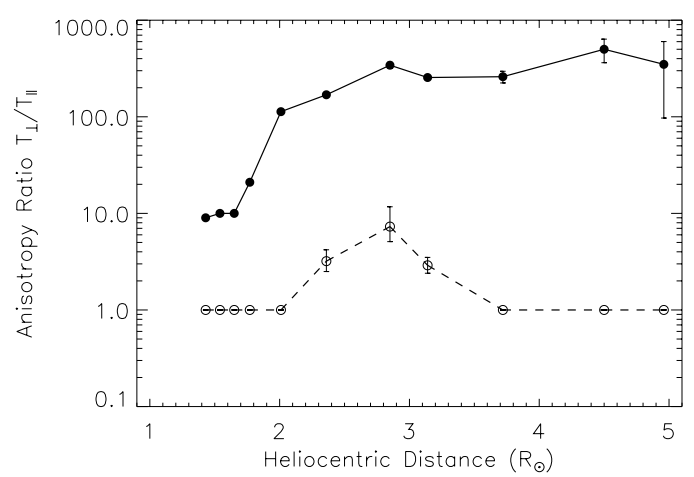

Fig. 4. Anisotropy ratio $T_{\perp} / T_{\|}$; open dots and dashed line correspond to the lowest degree of anisotropy allowed by the observations; full dots and solid line correspond to the maximum degree of anisotropy $\left(T_{\|, \min }=T_{\mathrm{e}}(r)\right)$.

and thus parallel oxygen temperature values, converge, within the uncertainties.

\section{Results}

The implication of the analysis is that by applying the diagnostic technique developed by Noci et al. (1987) to derive the fast wind speed, the observed ratios of the Doppler dimmed OVI 1037-1032 line intensities are consistent with a coronal model only when this is characterized by a significant anisotropy of the oxygen ion velocity distribution, at least between $2 R_{\odot}$ and $3.7 R_{\odot}$, just where the oxygen kinetic temperature reaches and maintains its maximum value of about $10^{8} \mathrm{~K}$. The lowest degree of the anisotropy ratio $T_{\perp} / T_{\|}$allowed by the measured O VI doublet intensity ratio is shown in Fig. 4 (open dots joined with the dashed line), where it is compared with the maximum anisotropy ratio $\left(T_{\mathrm{OVI}, \|}=T_{\mathrm{e}}\right)$ consistent with the data (full dots joined with the solid line).

The lowest degree of anisotropy increases from unity at $2 R_{\odot}$ to reach the maximum value of $T_{\perp} / T_{\|} \sim 7$ at $2.9 R_{\odot}$, and further out it decreases so that $T_{\perp} / T_{\|}$reaches unity again at $3.7 R_{\odot}$.

The degree of the anisotropy ratio, that is the oxygen temperature parallel to the magnetic field lines, is crucial in deriving the plasma outflow velocity. Due to the limitations imposed by the obtained anisotropy ratio results, it is possible to derive the 


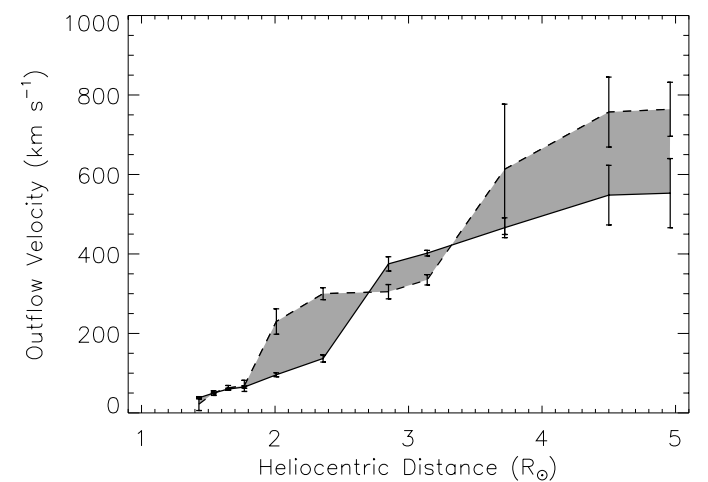

Fig. 5. OVI outflow velocity as a function of the heliocentric distance; dashed line corresponds to the lowest degree of anisotropy of the oxygen ion velocity distribution, whilst solid line corresponds to the maximum anisotropy; the gray area within the two curves represents the acceptance region for the outflow velocity of the oxygen component of the fast wind.

outflow velocity of the fast coronal wind only in a relatively wide acceptance region. The outflow speed results, derived by assuming the two extreme cases compatible with the data of anisotropy in the oxygen ion velocity distribution and, in turn, of the oxygen temperature parallel to the magnetic field lines, are plotted in Fig. 5.

The region within the two curves, inferred by assuming maximum and minimum anisotropy in the oxygen ion velocity distribution, defines the accuracy in deriving the velocity of the oxygen component of the fast wind due to the fact that the oxygen temperature parallel to the radial direction is not directly measured. The outflow velocity results clearly depend on the anisotropy degree of the velocity distribution of the oxygen ions above $1.8 R_{\odot}$, reaching $550 \mathrm{~km} \mathrm{~s}^{-1}$ and $760 \mathrm{~km} \mathrm{~s}^{-1}$ at $5 R_{\odot}$ in the case of maximum anisotropy or isotropy of the oxygen ion velocity distribution, respectively. At $5 R_{\odot}$ the most rapid expansion of the fast wind is found by assuming in the analysis the minimum anisotropy degree.

\section{Interpretation}

Previous literature clearly pointed to the incompatibility of the isotropic solution with the observational data beyond $2 R_{\odot}$ (Antonucci 1998; Kohl et al. 1998; Li et al. 1998; Dodero et al. 1998; Wilhelm et al. 1998; Antonucci et al. 2000; Cranmer et al. 1999). In these papers the issue of the degree of anisotropy has been addressed in the light of coronal heating models based on ion-cyclotron resonance scattering, which in principle can explain an extremely large anisotropy of the oxygen ion velocity distribution (e.g. Kohl et al. 1998; Cranmer et al. 1999). However, all these analyses consider the extended corona only out to $3.5 R_{\odot}$. In the present analysis we have extended the investigation of the oxygen velocity distribution beyond $3.5 R_{\odot}$, thus allowing us to measure the thickness of the coronal layer where the distribution is constrained to be anisotropic. Hence, this allows us to accurately identify the coronal region where energy is deposited and the ions are accelerated.

In the previous section, the upper and lower boundaries of the oxygen temperature anisotropy are derived from the observations. The largest degree of anisotropy allowed by the observational data (full dots and solid line in Fig. 4) over the full range of altitudes is not plausible as a possible physical interpretation, since it leads to an outflow speed of the expanding plasma that does not reach the observed asymptotic speed of

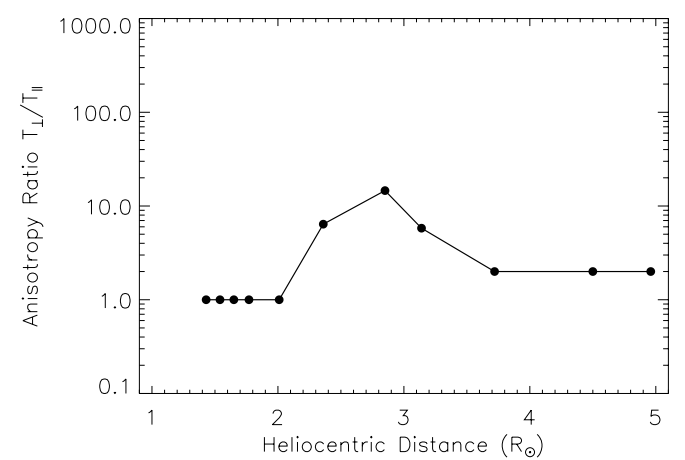

Fig. 6. Low anisotropy ratio $T_{\perp} / T_{\|}$representing a possible physical variation of anisotropy ratio with height.

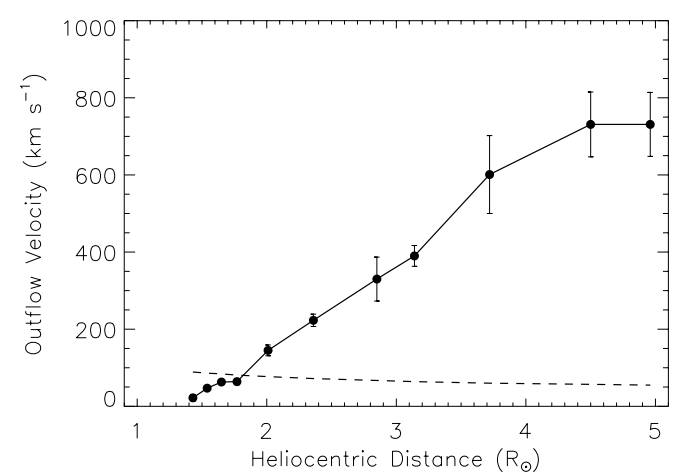

Fig. 7. OVI outflow velocity as a function of the heliocentric distance corresponding to the possible variation of anisotropy ratio with height shown in Fig. 6; the dot-dashed line shows the sonic velocity in a polar coronal hole.

$800 \mathrm{~km} \mathrm{~s}^{-1}$, which is achieved at about $8 R_{\odot}$ according to recent interplanetary scintillation (IPS) observations (e.g. Grall et al. 1995). An almost steady state regime with outflow velocity about $200 \mathrm{~km} \mathrm{~s}^{-1}$ smaller is instead reached beyond $4.5 R_{\odot}$ (solid line in Fig. 5). This consideration aids the interpretation of the data since an extremely large temperature anisotropy is quite difficult to be reproduced in models of energy deposition based on possible physical processes acting in the outer corona.

The outflow velocity derived by assuming the lowest degree of oxygen temperature anisotropy allowed by the observational data (open dots and dashed line in Fig. 4) does not meet the reasonable requirement that the speed of the expansion of the coronal plasma should maintain a positive acceleration with increasing heliocentric distance in the diverging open flux tubes above coronal holes, even if a value very close to the asymptotic speed $\left(760 \mathrm{~km} \mathrm{~s}^{-1}\right)$ is reached at $5 R_{\odot}$ (dashed line in Fig. 5). Hence, the variation of the anisotropy maintaining the minimum value allowed by the observed OVI doublet intensity ratio over the full range of altitudes cannot be valid throughout the observed region. However, it is sufficient to enhance the degree of anisotropy by a small factor (for instance 2) for heliocentric distances larger than $2 R_{\odot}$, in order to satisfy the condition that the acceleration remains positive throughout the outer corona. Hence, the anisotropy ratio is very close to the lowest one allowed by the observational data, although it does not reach unity beyond $3.7 R_{\odot}$ (Fig. 6). In this case, the two main requirements are achieved: the oxygen component of the solar wind maintains a positive and almost constant acceleration and it reaches an outflow velocity of $730 \mathrm{~km} \mathrm{~s}^{-1}$ at $5 R_{\odot}$, still close to the asymptotic speed (Fig. 7).

Thus, we conclude that very high anisotropies of the oxygen velocity distribution across the magnetic field, which are in 
contradiction with the existing models of coronal plasma acceleration, are indeed not necessary in order to explain the observations. These can be interpreted as being due to anisotropy degrees increasing up to 14 at maximum. The lower degrees of anisotropy found in this analysis can be more easily discussed and interpreted according to the existing models, thus solving the problems posed by the kinetic temperatures as high as $10^{8} \mathrm{~K}$ as invoked, for instance, by Cranmer et al. (1999).

On the other hand, the present results confirm the existence of the anisotropy found in the previous literature and rule out the conclusions by Raouafi \& Solanki $(2004,2006)$ implying that observational data can be reproduced without introducing an ion temperature anisotropy.

\section{Discussion}

In this section, the most recent ideas proposed for the plasma heating are discussed in the light of explaining the observed low degree of oxygen temperature anisotropy and the evolution of the velocity distribution of the ions, which departs from the biMaxwellian form at $2 R_{\odot}$ and approaches isotropy further out, at $3.7 R_{\odot}$ (Fig. 6).

In a collisionless plasma, the conservation of the ChewGoldberger-Low (CGL, Chew et al. 1956) adiabatic invariants leads to an evolution of the perpendicular and parallel component of the oxygen temperature as:

$T_{\perp} \propto B \quad$ and $\quad T_{\|} \propto \frac{n^{2}}{B^{2}}$.

In a coronal hole, in the approximation of a local spherical magnetic symmetry and a uniform expansion velocity, the oxygen temperature anisotropy as a function of the heliocentric distance is expressed by:

$\frac{T_{\perp}}{T_{\|}} \propto r^{-2}$.

Such evolution should be observed in the fast solar wind if there were no additional kinetic heating or acceleration processes. This is not the case at least in the region out to $2.9 R_{\odot}$, where the anisotropy ratio increases with height up to the maximum value $T_{\perp} / T_{\|} \sim 15$ (Fig. 6). Below $2 R_{\odot}$ the inferred Maxwellian ion velocity distribution implies that the energy is deposited preferentially across the magnetic field and the efficiency of the energy deposition varies with the heliocentric distance as $r^{2}$, in order to keep isotropy constant (cf. Eq. (5)). The transition from a Maxwellian to a bi-Maxwellian distribution, taking place from $2 R_{\odot}$ to $3.7 R_{\odot}$, indicates that the efficiency of the energy deposition across the magnetic field becomes higher in the region where the oxygen kinetic temperature reaches and maintains its maximum value of about $10^{8} \mathrm{~K}$. Beyond $2.9 R_{\odot}$, the evolution of the oxygen ion velocity distribution from a bi-Maxwellian to a quasi-Maxwellian configuration might be due to this geometrical effect, or other mechanisms may be involved.

The anisotropic temperature of oxygen ions $\left(T_{\perp}>T_{\|}\right)$is the main signature of the fast solar wind plasma heating and acceleration. The preferential heating of the oxygen wind component across the magnetic field, indicating an energy deposition acting in this direction in the outer corona, limits the range of possible physical processes responsible for heating the extended corona and points to the interpretation of the ion cyclotron heating mechanism, a collisionless wave-particle resonance (Kohl et al. 1998; Cranmer et al. 1999). If the frequency of the oscillating electric and magnetic fields due to Alfvén waves, propagating in the outer corona from the lower atmosphere, is the same as the cyclotron frequency of the ions, they absorb energy, thus increasing their velocity (and the orbital radius) but keeping a constant cyclotron frequency. Therefore, their perpendicular energy is increased at the expense of the wave energy. There are however still problems regarding how the Sun is capable of emitting Alfvén waves at much higher resonant frequencies (from $10 \mathrm{~Hz}$ to $\left.10^{4} \mathrm{~Hz}\right)$ than the ones actually emitted $(\lesssim 0.01 \mathrm{~Hz})$. High-frequency waves could be generated at lower heliocentric distances via reconnection events or in the extended corona via MHD turbulence cascade or ion cyclotron anisotropy instability.

Extremely high temperature anisotropies can be explained in this framework. On the other hand, low anisotropies can be explained by several processes including ion-cyclotron resonance. In this paper, we have shown that the observational data are likely to be consistent with an oxygen component of the fast solar wind that is only slightly anisotropic: numerous theoretical models are consistent with this latter result and in the following we describe some of them.

A turbulent cascade is a natural way of transforming fluctuations from a preexisting spectrum of low-frequency MHD waves to high-frequency Alfvén waves, resonant with oxygen ions (Cranmer \& van Ballegooijen 2003). If turbulent MHD displacements are smaller than the outer correlation length, the dissipation of Alfvénic turbulence in the extended corona will preferentially heat electrons along the magnetic field and there would be insufficient wave power at the ion cyclotron frequencies to heat oxygen ions. This could be a possible way of heating oxygen ions perpendicularly to the magnetic field, but without reaching extreme ion temperature anisotropy.

Kinetic models for ions have been developed in order to describe the wave heating and acceleration of the ions in the solar corona. The Vocks \& Marsch (2002) model includes waveparticle interactions and Coulomb collisions and it is based on reduced velocity distribution functions (VDFs). In the model of the extended corona, out to $3 R_{\odot}$, the plasma is dominated by wave-particle interactions and the oxygen ions reach very high temperatures: $T_{\perp}$ rises up to $10^{8} \mathrm{~K}$. The reduced VDFs of oxygen ions develop deviations from a local Maxwellian, but only a slight temperature anisotropy is obtained, $T_{\perp} / T_{\|} \sim 10$, throughout the range of the observation. This value is consistent with the results found in the present analysis $\left(\left(T_{\perp} / T_{\|}\right)_{\max } \sim 15\right)$, although the predicted evolution of the temperature anisotropy ratio with the increasing heliocentric distance is quite different.

Hybrid fluid-kinetic models, still based on cyclotron resonant heating of the ions, are characterized by wave-particle interaction, assuming the shape of the VDFs of the ions. Hybrid simulations by Li \& Habbal (2005) and Hellinger et al. (2005), which investigate resonant Alfvén wave heating of oxygen ions, are capable of reproducing the observed values and evolution of the anisotropy ratio described in Sect. 4 (Fig. 6). Although these simulations start from different assumptions for the plasma composition and the source of cyclotron resonance of the ions with the waves, both of them lead to the same conclusion: the heating of oxygen ions in the simulated plasma has two distinct stages. During the first one, when the system is stable with respect to the Alfvén ion cyclotron, $\mathrm{O}^{+5}$ ions are efficiently energized by ion cyclotron waves perpendicularly to the magnetic field lines but they are only slightly accelerated. The temperature anisotropy of $\mathrm{O}^{+5}$ rapidly increases and peaks at $10<\left(T_{\perp} / T_{\|}\right)_{\max }<22$, depending on the beta plasma value. This peak is experimentally observed in our analysis at $2.9 R_{\odot}$ (Fig. 6). After this initial phase, the system crosses the threshold of the oxygen cyclotron instability generated by the temperature anisotropy and the oxygen ion cyclotron resonance in the solar wind saturates, 
since only a small amount of energy of the propagating fluctuations is absorbed by $\mathrm{O}^{+5}$ ions. Therefore, in the second stage, ion cyclotron waves are not able to maintain a high temperature anisotropy, which decreases toward $T_{\perp} / T_{\|} \sim 2-3$, as inferred by the present analysis (Fig. 6). This anisotropy reduction is due to the fact that the temperature anisotropy instability leads to a parallel heating of oxygen ions by cyclotron waves, after the saturation. During this phase, oxygen ions are accelerated along the radial direction to the observed velocities (Li \& Habbal 2005).

An alternative theory to the dissipation of ion cyclotron resonant Alfvén waves was proposed by Lee \& Wu (2000): the fast shock heating. A fast shock mechanism for heating the heavy ions is able to reproduce satisfactorily the observed outflow velocity of the oxygen component of the fast solar wind and the evolution of the ion temperature anisotropy. Fast shocks, with an Alfvén Mach number $M_{A}<2$, may be generated by reconnection events associated with microflares and also by small-scale magnetic reconnection at the chromospheric level. For quasiparallel shocks, coronal heating occurs upstream of the shock front and it is due to the scattering by whistler waves and backstreaming ions. Since the generation of fast shocks is constant in time, oxygen ions are heated by several shocks during their outflow. The resulting dominant effect is ion heating perpendicularly to the magnetic field. In a low beta plasma filled with quasi-parallel subcritical shocks, the coronal heating leads to oxygen temperature anisotropies, which rise up to the maximum value of $\left(T_{\perp} / T_{\|}\right)_{\max } \sim 20$, consistent with the result inferred by the present analysis (Fig. 6). The Alfvén speed increases with the heliocentric distance to reach $V_{A} \sim 3500 \mathrm{~km} \mathrm{~s}^{-1}$ and it decreases further out; since in a low beta plasma heated by quasiparallel subcritical shocks the temperature anisotropy ratio and the Alfvén Mach number $M_{A}$ are anti-correlated, the oxygen ion anisotropy ratio decreases after reaching its maximum value toward lower values $\left(T_{\perp} / T_{\|} \sim 2\right)$, as expected by the observations (Fig. 6). The shock heating also leads to the observed outflow velocities for the oxygen component of the fast solar wind. The passing shocks directly accelerate the oxygen ions along their propagation direction, that is along the magnetic field lines since the fast shocks are quasi-parallel shocks. In addition, the temperature anisotropy generated by shock heating provides the oxygen ions with an outward field-aligned flow; due to the conservation of magnetic moment (Eq. 4), the perpendicular thermal energy $k T_{\perp}$ is converted to the radial outflow velocity as the ions move along the diverging coronal field lines. The resulting outflow velocity of the oxygen ions reaches $\sim 460 \mathrm{~km} \mathrm{~s}^{-1}$ at $3.5 R_{\odot}$, consistent with the results inferred in this paper (Fig. 6, $\left.u\left(3.5 R_{\odot}\right) \sim 520 \mathrm{~km} \mathrm{~s}^{-1} \pm 14 \%\right)$.

The energy required by the theory to accelerate the fast coronal wind to reach the asymptotic speed is provided above the sonic point, achieved at $1.8 R_{\odot}$ (Fig. 7), thus without a mass flux increase.

\section{Conclusions}

The lowest degree of anisotropy compatible with the observational data has been inferred throughout the observed outer corona. The velocity distribution of the oxygen ions has to be bi-Maxwellian at least in the coronal layer between $2 R_{\odot}$ and $3.7 R_{\odot}$

As the most plausible physical scenario, we infer that the velocity distribution of the $\mathrm{O}^{+5}$ ions is Maxwellian out to $2 R_{\odot}$ and further out it becomes bi-Maxwellian with a low anisotropy ratio twice the minimum allowed by the observations, increasing up to $\sim 14$ at $2.9 R_{\odot}$ and relaxing toward $\sim 2$ for larger heliocentric distances (open dots and dashed line out to $2.9 R_{\odot}$ and full dots and solid line further out in Fig. 6). The corresponding outflow velocity reaches $730 \mathrm{~km} \mathrm{~s}^{-1}$ at $5 R_{\odot}$ (a value close to the asymptotic speed) and the expansion of the coronal plasma occurs at a positive and almost constant acceleration (open dots and dashed line out to $2.9 R_{\odot}$ and full dots and solid line further out in Fig. 7).

Two types of theoretical models may satisfactorily explain the observed evolution of the velocity distribution of the oxygen ions and the inferred outflow velocity of the oxygen component of the fast solar wind. Although they are based on different processes of coronal plasma heating, both of them predict that the coronal heating leads to an oxygen temperature anisotropy increasing up to its maximum value $\left(T_{\perp} / T_{\|}\right)_{\max } \sim 10-20$ and then decreasing towards less values $T_{\perp} / T_{\|} \sim 2-3$. Hybrid fluidkinetic models by Li \& Habbal (2005) and Hellinger et al. (2005), based on cyclotron resonant heating of the oxygen ions, predict that the decrease of the anisotropy ratio starts when the system crosses the threshold of the oxygen cyclotron instability generated by the temperature anisotropy itself and thus when the oxygen ion cyclotron resonance saturates. On the other hand, the model proposed by Lee \& Wu (2000), based on a fast shock mechanism for heating the $\mathrm{O}^{+5}$ ions, predicts that in a low beta plasma filled by quasi-parallel subcritical shocks the reversal of the anisotropy ratio evolution takes place when the Alfvén speed crosses its maximum value and the heating perpendicular to the magnetic field hence becomes less efficient.

Acknowledgements. This work was supported by the Italian Space Agency (ASI) grants (I/035/05/0). Daniele Telloni is a PhD student with a fellowship of the National Institute for Astrophysics (INAF).

\section{References}

Antonucci, E. 1998, in A Crossroads for European Solar \& Heliospheric Physics, ESA SP-417

Antonucci, E. 2006, A\&ARv, in press

Antonucci, E., Giordano, S., \& Dodero, M. A. 2000, Adv. Space Res., 25, 1923 Antonucci, E., Dodero, M. A., Giordano, S., Krishnakumar, V., \& Noci, G. 2004, A\&A, 416, 749

Banaszkiewicz, M., Axford, W. I., \& McKenzie, J. F. 1998, A\&A, 337, 940

Chew, G. F., Goldberger, M. L., \& Low, F. E. 1956, Proc. R. Soc. London, Ser. A, 236, 112

Cranmer, S. R., Kohl, J. L., Noci, G. et al. 1999, ApJ, 511, 481

Cranmer, S. R., \& van Ballegooijen, A. A. 2003, ApJ 594, 573

Cranmer, S. R., Panasyuk, A. V., \& Kohl, J. L. 2005, in On the Incompatibility between UVCS/SOHO Observations of Polar Coronal Holes and Isotropic Oxygen Velocity Distributions, Eos Trans. AGU, 86(18), Joint Assembly Suppl., SP33A 02

David, C., Gabriel, A.-H., Bely-Dubau, F. et al. 1998, A\&A, 336, L90

Dodero, M. A., Antonucci, E., Giordano, S., \& Martin, R. 1998, Sol. Phys., 183, 77

Grall, R. R., Coles, W. A., Klinglesmith, M. T. 1995, in Observations of the solar wind speed near the sun, California Univ., Int. Solar Wind 8 Conf., 61

Guhathakurta, M., Fludra, A., Gibson, S. E., Biesecker, D., \& Fisher, R. 1999, JGR, 104(A5), 9801

Hellinger, P., Velli, M., Trávnícek, P., et al. 2005, JGR, 110, A12109

Kohl, J. L., Noci, G., Antonucci, E., et al. 1998, ApJ, 501, L127

Kohl, J. L., Noci, G., Cranmer, S. R., \& Raymond, J. C. 2006, A\&ARv, 13, 31

Lee, L. C., \& Wu, B. H. 2000, ApJ, 535, 1014

Li, X., \& Habbal, S. R. 2005, JGR, 110, A10109

Li, X., Habbal, S. R., Kohl, J. L., \& Noci, G. 1998, ApJ, 501, L133

Noci, G., Kohl, J. L., \& Withbroe, G. L. 1987, ApJ, 315, 706

Raouafi, N. E., \& Solanki, S. K. 2004, A\&A, 427, 725

Raouafi, N. E., \& Solanki, S. K. 2006, A\&A, 445, 735

Schwenn, R. 1990, in Physics of the Inner Heliosphere, ed. R. Schwenn, \& E. K.

Marsch (Springer Verlag), 1, 99

Telloni, D., Antonucci, E., \& Dodero, M. A. 2007, A\&A, 472, 299

Vocks, C., \& Marsch, E. 2001, Geophys. Res. Lett., 28, 1917

Wilhelm, K., Marsch, E., Dwivedi, B. N., et al. 1998, ApJ, 500, 1023 